\title{
Neutron resonance capture analysis and applications
}

\author{
H. Postma, ${ }^{1}$ R. C. Perego, ${ }^{1}$ P. Schillebeeckx, ${ }^{2}$ P. Siegler, ${ }^{2}$ A. Borella ${ }^{2}$ \\ ${ }^{I}$ IRI, University of Technology, Mekelweg 15, 2629 JB Delft, The Netherlands \\ 2 JRC-IRMM, Retieseweg, Geel, B-2440, Belgium
}

(Received April 13, 2006)

In this paper we discuss the use of neutron resonances to determine the elemental composition of objects non-destructively. Resonances are observed from about 1 to $3000 \mathrm{eV}$ by the time-of-flight technique using the pulsed neutron source of the GELINA facility in Geel (Belgium). Prompt capture $\gamma$-radiation is used to signal capture events. Some aspects of the neutron resonance capture analysis (NRCA) are compared with prompt gamma neutron activation analysis (PGNAA).

\section{Introduction}

It has recently been shown that resonances occurring in neutron capture cross sections as a function of neutron energy are very suitable to analyze the elemental composition of materials and objects. This is a new analytical method developed at the GELINA facility in Geel (Belgium) as a joint project of the Interfaculty Reactor Institute of the Delft University of Technology and the EU Joint Research Centre IRMM, and is called "neutron resonance capture analysis" (NRCA). Energies of captured neutrons are determined with the time-offlight method (TOF) using the pulsed neutron source of GELINA. The machine pulse and the signal obtained by detecting prompt $\gamma$-radiation are the time markers for the TOF of each captured neutron. Prompt $\gamma$-radiation is accepted in a wide energy range. Together with the fact that in most cases capture is followed by a number of $\gamma$ rays in cascade, it is possible to achieve large detection efficiencies. In this novel method energies of individual prompt $\gamma$-rays are not essential.

NRCA is fully non-destructive, it can be used to determine the bulk composition of objects without preparation or sample taking. It results in a negligible residual activation. It is applied in a number of studies of bronze artefacts. ${ }^{1-3}$ Other applications are considered and tested. 4

In prompt gamma activation analysis (PGNAA), developed to a high level by Gábor MOLNÁR and his group at the Budapest reactor and applied at several other institutes, energies of prompt $\gamma$-rays are used to recognize elements. ${ }^{5,6}$ Some aspects of NRCA and PGNAA will be compared in this paper.

\section{Experimental}

\section{The GELINA facility}

The GELINA facility in Geel is a linear electron accelerator with maximum electron energy of $150 \mathrm{MeV}$ and maximum beam power of $10 \mathrm{~kW}$ with a pulse rate up to $800 \mathrm{~Hz}$. The pulse width after the bunching magnet is as short as $1 \mathrm{~ns}$. Neutrons are produced when these electrons hit a uranium target. To enhance the neutron intensity in the low energy domain, two waterfilled Be-containers (4 cm thick) serve as partial moderators. They are placed just above and below the uranium target. The neutron fluence rate at distance $L$ (in $\mathrm{m})$ from the production source is in good approximation:

$$
1.6 \cdot 10^{6} L^{-2} \cdot E^{-0.92} \mathrm{~s}^{-1} \cdot \mathrm{eV}^{-1} \cdot \mathrm{cm}^{-2}
$$

with $E$ in $\mathrm{eV}$. We have used flight-path lengths of approximately 14 and $28 \mathrm{~m}$ with the beam collimated at the sample position to $6.5 \mathrm{~cm}$. In recent experiments we used two cylindrical $\mathrm{C}_{6} \mathrm{D}_{6}$ scintillation detectors, $7.6 \mathrm{~cm}$ thick and $12.6 \mathrm{~cm}$ in diameter. They are placed at a distance of $7 \mathrm{~cm}$ from the centre of the beam. The time of flight is registered with a timecoder with basic clock period of $0.5 \mathrm{~ns}$. Appropriate channel widths and regions are chosen to cover the time between the machine pulses. A TOF spectrum can be converted to an energy spectrum by:

$$
E_{n}=\left(72.298 \frac{L}{T+T_{0}}\right)^{2}
$$

where $E_{\mathrm{n}}$ is the neutron energy in $\mathrm{eV}, L$ is in $\mathrm{m}$, and $T$ the TOF in $\mu \mathrm{s} . T_{0}$ is a constant representing a small time off-set within the experimental system.

Already during data-taking resonance peaks can be recognized, thus providing a quick and qualitative analysis of the elemental composition.

\section{Contents and shapes of the resonance peaks}

For the quantitative analysis we need to determine the total number of counts in the resonance peaks. Often these numbers can be obtained by summing the contents of channels covering such peaks, and subtracting the backgrounds obtained from regions on both sides of the peaks. In the case of overlapping resonances or if a bump occurs at the high energy side of a resonance due 
to scattering followed by capture, it is necessary to do peak-fit analysis. The basic shape of a resonance is given by the Breit-Wigner expression with full width at half maximum, $\Gamma$, which is the sum of the neutron and radiation widths:

$$
\Gamma=\Gamma_{n}+\Gamma_{\gamma}
$$

Due to Doppler and instrumental effects resonances will be broadened. This changes the original Lorentzianlike shape of a resonance to a Gaussian shape with wings, which may still be Lorentzian. We have fitted resonances with Gaussian and Voigt shapes. The latter is the sum of a Gaussian and a Lorentzian function. If there is strong absorption at a resonance this is not an appropriate approach. Then, more sophisticated fitting procedures (REFIT, ${ }^{7}$ SAMMY) ${ }^{8}$ must be considered.

\section{Neutron resonance capture analysis}

The way we have been analyzing the data in order to get quantitative information is by taking ratios of the contents, $N\left(E_{\mathrm{I}}\right)$ and $N\left(E_{\mathrm{II}}\right)$, of the resonance peaks of two elements, I and II, of the object. This is compared with the same ratio from a calibration sample of known composition. That is, the weight ratio, $W_{\mathrm{I}} / W_{\mathrm{II}}$, of the object is related to the weight ratio of the calibration sample by:

$$
\left(\frac{W_{\mathrm{I}}}{W_{\mathrm{II}}}\right)_{o b}=\left(\frac{N\left(E_{\mathrm{I}}\right)}{N\left(E_{\mathrm{II}}\right)}\right)_{o b} \cdot\left(\frac{N\left(E_{\mathrm{II}}\right)}{N\left(E_{\mathrm{I}}\right)}\right)_{c a} \cdot R \cdot\left(\frac{W_{\mathrm{I}}}{W_{\mathrm{II}}}\right)_{c a}
$$

During traversing the object, the neutron fluence will be reduced at the resonances due to capture and scattering. This is known as the self-shielding effect for which the experimental number of counts must be corrected. Equation (2) contains the factor $R$, in which four self-shielding factors are compounded. Details of the way we handle the self-shielding can be found in Reference 9. We prefer to use resonances with small self-shielding effects. For major components the weaker resonances should be used. Elements have often several useful resonances in the energy range of about 1 to about $3000 \mathrm{eV}$, which is covered with the current TOF system.

\section{Resonance sensitivity}

The number of counts in a resonance peak depends on the neutron fluence, the so-called resonance capture area $\left(g \Gamma_{n} \Gamma_{\gamma} / E_{0} \Gamma\right)$, and the isotope abundance, $a$. The neutron fluence rate at the GELINA facility and similar machines is approximately inverse by proportional to the energy. This leads to the following definition of a suitable sensitivity factor, $S$ :

$$
S=\frac{g \Gamma_{n} \Gamma_{\gamma}}{\Gamma} \cdot \frac{a}{E_{0}^{2}}
$$

where $g$ is a spin-dependent statistical factor. The $S$ factor varies over a very wide range, even between resonances of the same isotope. It is clear from Eq. (3) that $S$ decreases rapidly with increasing energy. Therefore, the sensitivity to detect elements can be very large if they have resonances at a few eV.

\section{Detection efficiency for capture events}

In the first NRCA experiments we have used four BGO-scintillation detectors. Recently we switched to two $\mathrm{C}_{6} \mathrm{D}_{6}$ detectors. They have lower $\gamma$-detection efficiencies. However, they have two important advantages: (1) excellent time resolution, and (2) very low sensitivity for neutrons. Therefore, these detectors have a much lower background. ${ }^{4}$ The $\gamma$-energy resolution of $\mathrm{C}_{6} \mathrm{D}_{6}$ detectors is poor but this is not a disadvantage since we need only a signal to know that capture has occurred. Our detection system is set to accept all $\gamma$-rays between about $0.3 \mathrm{MeV}$ and $10 \mathrm{MeV}$.

The $\gamma$-emission after neutron capture is largely a statistical process with usually a very large number of intermediate levels. The number of steps, $v$, in a $\gamma$ cascade to the ground state can be as large as 6 . The sum of their energies $\left(E_{i}\right)$ is equal to the capture excitation energy $\left(E_{\mathrm{C}}\right)$. The probability $p_{\text {event }}$ for detecting a capture event is given by:

$$
p_{\text {event }}=1-\prod_{i=1}^{v}\left(1-p_{i}\right)
$$

where $p_{\mathrm{i}}$ are the detection efficiencies for the $\gamma$-rays in a cascade. Since there are many cascades possible, the actual detection efficiency, $\varepsilon_{\text {capt }}$, is a weighted average of $p_{\text {event }}$. If wanted, capture detection efficiencies can be calculated with a Monte Carlo procedure using capture $\gamma$-spectra simulated for instance with the method developed by ZANINI et al. ${ }^{10}$ It should be noted that these efficiencies will be different for different nuclei, even for resonances of the same isotope. Under the simplifying assumption of a fixed number of steps, $v$, all with the same energy $E_{\mathrm{C}} / v$, Eq. (4) transforms to:

$$
\varepsilon_{\text {capt }}=1-(1-p)^{v}
$$

If $p$ for the cascade $\gamma$-rays is small, $\varepsilon_{\text {capt }}$ is, as expected, approximately equal to $v p$. For the two $\mathrm{C}_{6} \mathrm{D}_{6}$ detectors the capture detection efficiency varies from about 0.06 to 0.29 for $v=2$ to 6 . Currently we are working on the realization of an array of ten $\mathrm{C}_{6} \mathrm{D}_{6}$ detectors placed in a dodecaeder arrangement with two remaining, opposite positions for beam entrance and exit. This system will have detection efficiencies from about 0.36 to 0.9 for $v=2$ to 6 . 


\section{Results}

Recently a project was started to study the elemental composition of prehistoric copper-based axes, among them five axes on loan from the National Museum of Antiquity in Leiden (NL). Figure 1 shows the TOF spectrum of one of these axes. A small section of one of the spectra, converted to the energy scale, is shown in
Fig. 2 together with the result of a fitting procedure. If possible we use several pairs of resonances to obtain weight ratios. For instance, for copper we used resonances at 230, 650, 994 and $1363 \mathrm{eV}$, for tin resonances at 33.8 and $111 \mathrm{eV}$. This provides eight values for the $\mathrm{Sn} / \mathrm{Cu}$ weight ratio, which have different self-shielding effects. If the analysis is carried out properly, the corrected ratios should be close (Fig. 3).

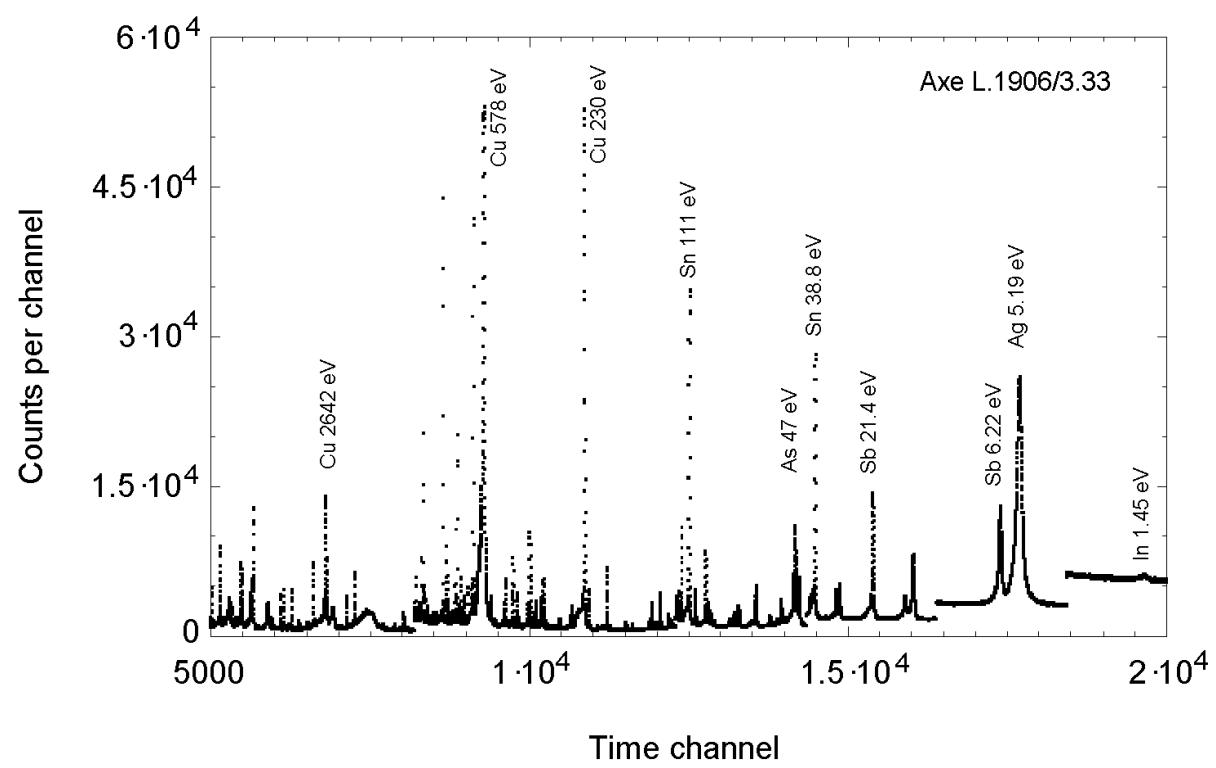

Fig. 1. TOF spectrum as a function of time channels of one of the prehistoric copper-alloy axes on loan from the National Museum of Antiquity, Leiden (NL). Energies of several resonances of interest are indicated

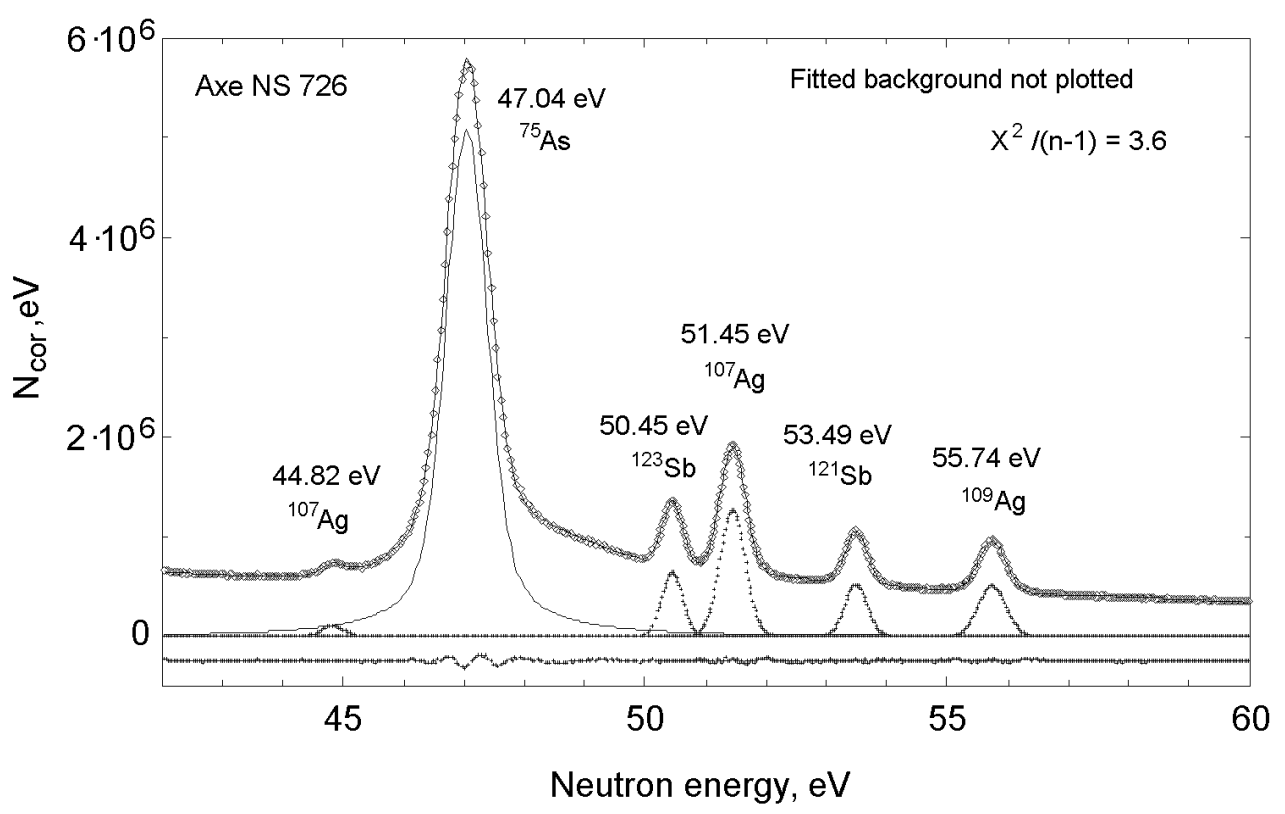

Fig. 2. Part of the energy spectrum of axe (NS 726) with the $47 \mathrm{eV}$ resonance of ${ }^{75} \mathrm{As}$ and smaller peaks of silver and antimony isotopes. The $47 \mathrm{eV}$ peak has a bump at the high-energy side. The line through the data points is fitted with a Voigt curve for the $47 \mathrm{eV}$ peak and with Gaussian functions for the smaller peaks and bump. The bottom line shows the residuals of the fitted curve 


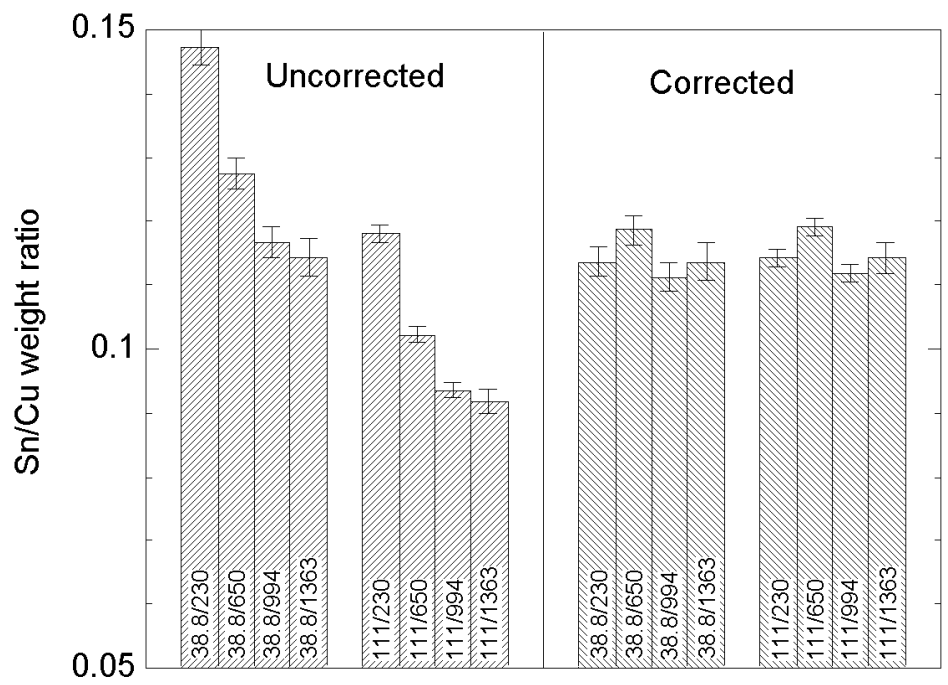

Fig. 3. Weight ratios of $\mathrm{Sn}$ to $\mathrm{Cu}$ of one of the axes (1931/2.74) determined for eight pairs of resonances without self-shielding corrections, and corrected. For copper resonances at 230, 650, 994 and $1363 \mathrm{eV}$ are used for tin the 33.8 and $111 \mathrm{eV}$ resonances. Statistical errors are given. The corrected ratios are in good agreement with each other

Table 1. Ratios of elements with respect to copper of five axes with the machine running at $800 \mathrm{~Hz}$ except for axe 1906/3.33 with the machine at $100 \mathrm{~Hz}$. For all measurements the $28 \mathrm{~m}$ flight path has been used

\begin{tabular}{|c|c|c|c|c|c|}
\hline Element ratio & $1906 / 3.33$ & NS 726 & NS 727 & NS 750 & $1931 / 2.74$ \\
\hline $\mathrm{Sn} / \mathrm{Cu}$ & $0.1513 \pm 0.0023$ & $0.0105 \pm 0.0002$ & $0.0758 \pm 0.0012$ & $0.1572 \pm 0.0027$ & $0.1122 \pm 0.0017$ \\
\hline $\mathrm{As} / \mathrm{Cu}$ & $0.00198 \pm 0.00004$ & $0.0176 \pm 0.0002$ & $0.0269 \pm 0.0005$ & $0.00086 \pm 0.00006$ & $0.0119 \pm 0.0002$ \\
\hline $\mathrm{Sb} / \mathrm{Cu}$ & $0.00274 \pm 0.00004$ & $0.0325 \pm 0.0005$ & $0.0308 \pm 0.0005$ & $0.00012 \pm 0.00002$ & $0.0249 \pm 0.0004$ \\
\hline $\mathrm{Ag} / \mathrm{Cu}$ & $0.00214 \pm 0.00036$ & $0.0146 \pm 0.0003$ & $0.0243 \pm 0.0005$ & $0.00050 \pm 0.00002$ & $0.0262 \pm 0.0005$ \\
\hline $\mathrm{Co} / \mathrm{Cu}$ & & & $0.0017 \pm 0.0001$ & & $0.0058 \pm 0.0002$ \\
\hline $\mathrm{Fe} / \mathrm{Cu}$ & & & & $0.0770 \pm 0.0037$ & $0.0072 \pm 0.0006$ \\
\hline $\mathrm{Pb} / \mathrm{Cu}$ & $0.17 \pm 0.02$ & & & & \\
\hline $\mathrm{In} / \mathrm{Cu}$ & $18 \mathrm{ppm} \pm 6$ & o.o.r. & o.o.r. & o.o.r. & o.o.r. \\
\hline
\end{tabular}

o.o.r.: Out of range.

In Table 1 the weight ratios of the axes with respect to copper are quoted. The axes show rather different compositions. Four have considerable amounts of tin with ratios to copper from 7 to $15 \%$. Normally bronze artefacts have fractions of a percent of $\mathrm{As}, \mathrm{Sb}$ and $\mathrm{Ag}$. That is true for two of these axes (1906/3.33 and NS750), the first is clearly a leaded bronze. The other three (NS726, NS727 and 1931/2.74) have percentage amounts of these elements, which is curious and points to a different production technique.

\section{Discussion and conclusion}

NRCA and PGNAA are closely related methods. Both use the prompt capture $\gamma$-radiation, NRCA to obtain a capture resonance spectrum as function of neutron energy, and PGNAA to determine elements on the basis of prompt $\gamma$-ray energies. Therefore, they need different kinds of neutron beams. NRCA requires a pulsed, white beam and depends, therefore, on accelerators. PGNAA requires a (sub)thermal, steady state beam. The neutron fluence at the GELINA facility is low compared to the beam used by MOLNÁR's group. However, the lower fluence for our NRCA experiments is well compensated by the large detection efficiencies compared to that of the HP-Ge detector plus BGO shield used by MOLNÁR et al. The lower neutron fluence rate and the use of a $\mathrm{Cd}$ filter to remove neutrons below $\approx 0.7 \mathrm{eV}$ from the beam means that a negligible residual activation is very quickly reached at our facility.

In both methods it is possible to get a qualitative determination of the composition during data taking. Quantitative analysis can be done directly thereafter. Both methods determine bulk compositions of objects. The sensitivity to detect elements depends to a large extent on the $S$-factors in the case of NRCA and on the so-called $k_{0}$-factors for PGNAA. ${ }^{11}$ Both of these factors show large variations. Certain elements are better detected with NRCA, others with PGNAA. The latter is better for the lighter elements. NCRA has often a larger sensitivity for elements with resonances of a few $\mathrm{eV}$. 


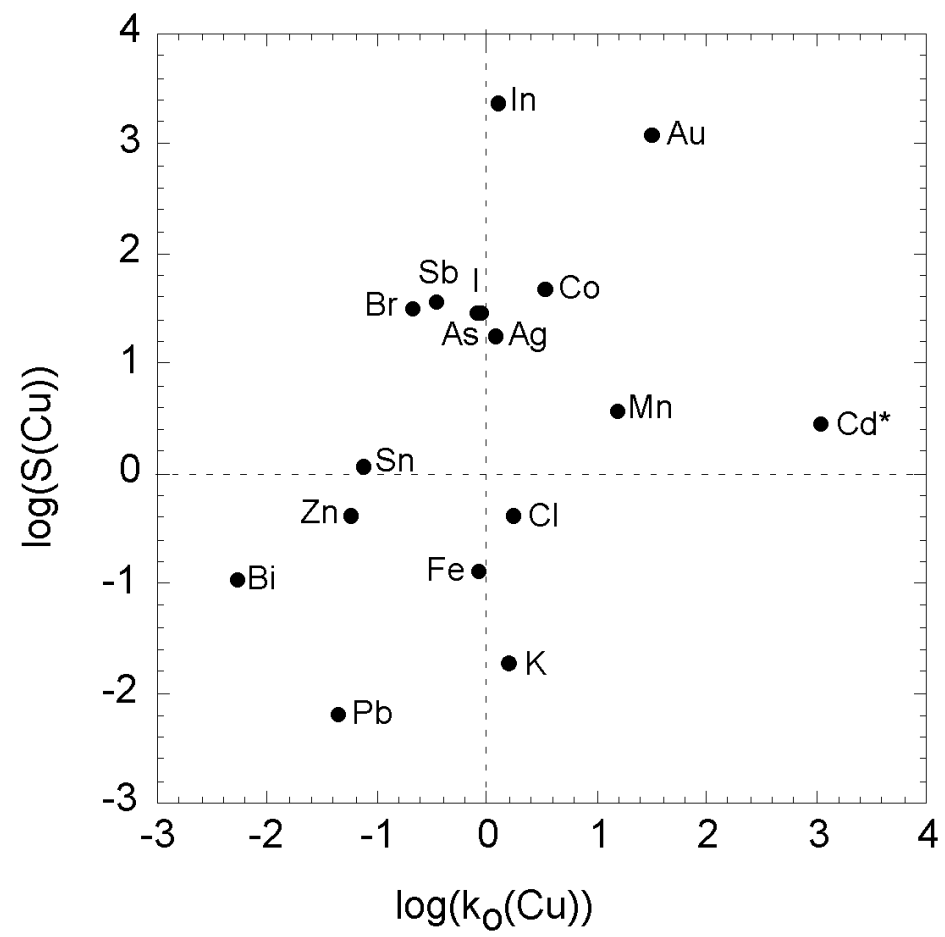

Fig. 4. A comparison of $S$ - and $k_{0}$-factors normalized to their copper values in a log-log plot for several elements encountered in studies of copper-based artefacts. * For Cd the $k_{0}$-value may be different when measured in different neutron beams due to the non-1/v behavior of the cross section ${ }^{11}$

Figure 4 gives a comparison of $S$ - and $k_{0}$-factors normalized to their copper values, on a log-log plot for elements encountered in various studies of copper-based artefacts. The figure shows that some elements can be better detected as trace elements by NRCA, and others by PGNAA. An interesting case is indium, which is a new trace element in bronze artefacts, since it can be very well detected by NRCA.

As a conclusion it might be stated that a combination of PGNAA and NRCA would be very valuable for the study of artefacts and possibly also for other applications.

Marie-Frans OORSOUw of the NMA very kindly arranged the loan of the prehistoric axes. We very much like to thank Wim MONDELAERS and his staff for the very skilful operation of the Linac of IRMM.

\section{References}

1. H. Postma, M. Blahuw, P. Bode, P. Mutti, F. Convi, P. SIEGLER, J. Radioanal. Nucl. Chem., 248 (2001) 115.

2. M. Blahuw, H. Postma, P. Mutti, Nucl. Instr. Meth. Phys. Res., A505 (2003) 508.

3. H. Postma, P. Schillebeeckx, R. B. Halbertsma, Archaeometry, 46 (4) 635-646 (2004).

4. R. C. Perego, H. Postma, M. Blaauw, P. Schillebeeckx, A. BoRella, J. Radioanal. Nucl. Chem., 271 (2007) 89.

5. G. Molnár, T. Belgya, L. Dabolci, B. Fazekas, Zs. Révay, Á. VERES, I. BIKIT, Z. KISS, J. ÖsTÖR, J. Radioanal. Nucl. Chem., 215 (1997) 111

6. Zs. Kastovszky, Zs. Révay, T. Belgya, G. L. Molnár, J. Radioanal. Nucl. Chem., 244 (2000) 379.

7. M. C. Moxon, J. B. BrisLand, Technical Report Harwell Laboratory, CBNM/ST/90-131/1, 1990.

8. N. M. LARSON, ORNL/TM-9179/R5, 2000.

9. H. Postma, M. Blahuw, P. Schillebeeckx, G. Lobo, R. Halbertsma, A. J. Nijboer, Czechosl. J. Phys., 33 (2003) A233.

10. L. Zanini, F. Corvi, H. Postma, F. Becvár, M. Krticka, J. HonZÁtKo, I. TOMANDL, Phys. Rev., C68 (2003) 014320-1/13.

11. Zs. Révay, G. L. MolnáR, T. Belgya, Zs. Kasztovszky, R. B. Firestone, J. Radioanal. Nucl. Chem., 244 (2000) 383. 